\title{
CAMBIOS MORFOLÓGICOS EN LA MANDÍBULA DURANTE LA ONTOGENIA: UN APORTE DESDE LA HISTOLOGÍA Y LA MORFOMETRÍA GEOMÉTRICA
}

\author{
Natalia Brachetta Aporta1', Paula N. Gonzalez ${ }^{1,2}$, Valeria Bernal ${ }^{1}$ y Cayetana Martinez-Maza ${ }^{3 *}$ \\ ${ }^{I}$ División Antropología. Facultad de Ciencias Naturales y Museo. Universidad Nacional de La Plata. CONICET. La Plata. Argentina \\ ${ }^{2}$ Instituto de Genética Veterinaria. Facultad de Ciencias Veterinarias UNLP-CCT. CONICET. La Plata. Argentina \\ ${ }^{3}$ Departamento de Paleobiología. Museo Nacional de Ciencias Naturales (CSIC). Madrid. España
}

PALABRAS CLAVE crecimiento; modelado óseo; forma y tamaño

\begin{abstract}
RESUMEN El análisis de la morfología craneofacial durante la ontogenia es una línea central en los estudios evolutivos, del desarrollo y biomédicos. El complejo craneofacial ha sido estudiado desde dos perspectivas: el análisis de la forma y el tamaño mediante morfometría lineal y geométrica, y el estudio de los procesos subyacentes mediante el análisis histológico de la superficie del hueso. El objetivo de este trabajo es integrar estas dos líneas para analizar los cambios morfológicos de la mandíbula y ofrecer un modelo de crecimiento que dé cuenta de los mismos. Se analizó una muestra de mandíbulas humanas de individuos subadultos y adultos de procedencia europea. La variación en forma fue registrada a partir de coordenadas de landmarks y análisis de regresión sobre el tamaño. Los campos de formación y
\end{abstract}

reabsorción ósea fueron identificados sobre réplicas de alta resolución de la superficie ósea. Los resultados indican que la variación a escala anatómica descrita mediante técnicas morfométricas fue, en líneas generales, concordante con la inferida a partir de la distribución de las áreas de modelado óseo. Sin embargo, cambios importantes como los movimientos de rotación de la mandíbula con el crecimiento sólo pudieron detectarse a través del análisis morfométrico. Asimismo, ambos tipos de datos mostraron diferencias en las direcciones de cambio inferidas para la sínfisis mandibular. Este trabajo señala la importancia de integrar datos histológicos y morfométricos para comprender los patrones y procesos de cambio morfológico en la ontogenia. Rev Arg Antrop Biol 18(2), 2016. doi:10.17139/raab.2016.0018.02.06

\section{KEYWORDS growth; bone modeling; size and shape}

\begin{abstract}
The analysis of craniofacial morphology during ontogeny has a central role in evolutionary, developmental, and biomedical studies. The craniofacial complex has been studied from two main perspectives: the analysis of its shape and size by means of linear and geometric morphometrics, and the study of underlying processes through histological analysis of the bone surface. The aim of this work is to integrate these two lines of evidence to analyze the morphological changes of the mandible and provide a growth model that can account for such changes. We analyzed a sample of human mandibles from subadult and adult individuals of European origin. Shape changes were analyzed using landmark coordinates and regressions of shape coordinates on size. Areas of
\end{abstract}

bone formation and resorption were identified on high-resolution replicas of the bone surface. The results indicate that variation at the anatomical scale, as described by morphometric techniques, is broadly consistent with that inferred from the distribution of the areas of bone modeling. However, important changes such as growth-related rotational movements of the mandible could only be detected through morphometric analysis. Also, both types of data showed differences in the directions of change inferred for the mandibular symphysis. This work highlights the importance of integrating histological and morphometric data to understand the patterns and processes of morphological change in ontogeny. Rev Arg Antrop Biol 18(2), 2016. doi:10.17139/raab.2016.0018.02.06
El análisis morfológico de estructuras óseas complejas a lo largo de la ontogenia es central en estudios evolutivos, del desarrollo y biomédicos (Mitteroecker et al., 2004; Halazonetis, 2007; Wilson et al., 2015; entre otros). Durante la ontogenia las estructuras anatómicas aumentan en tamaño como consecuencia del crecimiento y cambian en forma debido tanto al crecimiento diferencial de los elementos anatómicos (i.e. alometría) como a modificaciones en la posición espacial de los mismos a través del tiempo. En líneas generales, los cambios ocurridos pueden ser descritos a través de dos propiedades, la magnitud y dirección del cre-

Financiamiento: PIP 0428 - CONICET. PI: 11/N742, 11/ N710- UNLP. Universidad Nacional de La Plata (M157).

*Correspondencia a: Cayetana Martínez Maza. Departamento de Paleobiología. Museo Nacional de Ciencias Naturales (CSIC). Jose Gutierrez Abascal 2. 28006 Madrid. España. E-mail: martinezmaza.cayetana@gmail.com

Recibido 17 Marzo 2015; aceptado 23 Julio 2015

doi:10.17139/raab.2016.0018.02.06 
cimiento, que en conjunto determinan la morfología de las estructuras óseas en cada etapa ontogenética (Enlow, 1982). La variación en tamaño y forma observable en la escala anatómica es una manifestación de procesos que operan, en parte, a escalas de organización jerárquica menores. En particular, los procesos que tienen lugar a escala tisular y celular resultan relevantes para comprender el origen y mantenimiento de los patrones observables a nivel morfológico (Atchley y Hall, 1991; Hall, 2003). La complejidad de estos procesos puede ser interpretada en el marco teórico de la biología craneofacial establecida por Enlow (1963, 1966), Enlow y Hans, (1996) y Moss (1970, 1997a-d) que permite establecer un modelo de crecimiento de la estructura anatómica, considerando direcciones de cambio específicas y teniendo en cuenta los desplazamientos primarios (i.e. poblaciones celulares locales) y secundarios de áreas puntuales como consecuencia de su interacción con otros componentes del sistema craneofacial en el desarrollo y las demandas funcionales de los tejidos blandos circundantes.

El desarrollo de técnicas de morfometría geométrica hacia la década de 1990 ha proporcionado un marco matemático especialmente adecuado para describir cuantitativamente la magnitud y dirección de los cambios morfológicos que ocurren hasta alcanzar la conformación adulta (Adams et al., 2004; Mitteroecker y Gunz, 2009). Estas técnicas -basadas en coordenadas de puntos anatómicos- preservan la geometría de las estructuras, y por lo tanto, permiten describir y visualizar variaciones en la posición espacial de los elementos anatómicos y realizar inferencias acerca de las direcciones de cambio. Asimismo, la información acerca de la forma puede ser extraída y analizada independientemente de la variación alométrica asociada al tamaño. Sin embargo, los estudios basados en análisis morfogeométricos exclusivamente resultan limitados para explicar cómo se generan los cambios anatómicos en términos de los mecanismos del desarrollo que operan a escalas de organización jerárquica menores.

En este sentido, el análisis histológico de estructuras asociadas al modelado óseo puede contribuir al estudio de los procesos tisulares involucrados en los cambios en tamaño y forma de las estructuras morfológicas complejas durante el crecimiento de un individuo (Bastir et al., 2007). En particular, las diferencias en la duración y tasa de actividad de los osteoblastos y osteoclastos, así como en la localización, extensión y distribución espacial de las áreas formación y reabsorción ósea modelan el cambio en tamaño y forma de una morfología dada durante la ontogenia (Enlow, 1966). La distribución de las áreas de actividad celular representa un patrón de modelado óseo que contribuye a establecer direcciones de crecimiento del hueso, considerando que las superficies de formación indican el sentido del crecimiento óseo mientras que las superficies de reabsorción indican la dirección de crecimiento contraria (Enlow y Hans, 1996).

Hasta el presente las aproximaciones metodológicas morfométrica e histológica han sido empleadas de manera independiente en los estudios morfológicos. Sólo tres estudios han abordado el análisis conjunto de datos histológicos y morfogeométricos en el cráneo: uno en poblaciones humanas (Brachetta Aporta et al., 2014) $\mathrm{y}$ dos en primates no humanos (O'Higgins y Jones, 1998; Friedline et al., 2014). En este sentido, en el presente trabajo se propone realizar de manera exploratoria el análisis conjunto de los patrones de cambio morfológico durante la ontogenia usando técnicas de la morfometría geométrica y los patrones de modelado óseo obtenidos a partir del análisis histológico empleando como sistema modelo la mandíbula humana. En particular, se evaluará: a) la concordancia en las trayectorias de cambio a lo largo de la ontogenia en humanos (desde la erupción del primer molar hasta la etapa adulta) inferidas a partir de datos obtenidos a escala anatómica e histológi$\mathrm{ca}, \mathrm{y}$ b) la correspondencia en la variación interindividual a ambas escalas. Los resultados de este estudio contribuirán a discutir en qué medida los patrones de modelado óseo covarían con los cambios anatómicos, así como la posibilidad de efectuar inferencias acerca de los procesos involucrados en el cambio morfológico con base en los patrones de modelado óseo.

\section{MATERIAL Y MÉTODOS}

La muestra analizada corresponde a mandíbulas de individuos de ambos sexos cuyas edades se encuentran entre 7 y 48 años. Los mis- 
mos proceden de entierros del cementerio "da Conchada" de Coimbra (Portugal) datados entre fines del siglo XIX-principios del siglo XX (Rocha, 1995) y forman parte de la colección de Esqueletos Identificados del Departamento de Antropología de la Universidad de Coimbra (Portugal). La totalidad de los individuos cuentan con información acerca del sexo, la edad, la ancestría y las causas de muerte. A los fines de este estudio se asume que no hay diferencias morfológicas significativas entre los individuos atribuibles a la procedencia geográfica y la cronología.

\section{Análisis morfométrico}

El análisis morfométrico se efectuó sobre 87 mandíbulas. Para este análisis los individuos fueron agrupados en dos grandes categorías etarias: subadultos (incluyendo desde la menor edad representada en la muestra, 7 años, hasta los 18 años; $n=28$, femeninos $=16$, masculinos $=12)$ y adultos (18-48 años; $n=59$, femeninos $=31$, masculinos $=28$ ). La morfología se describió mediante 16 landmarks tridimensionales obtenidos con un Microscribe G2X (Fig. 1, Tabla 1 Apéndice Online 1). Las coordenadas de landmarks fueron superpuestas mediante el método Procrustes por mínimos cuadrados (Rohlf y Slice, 1990).

Con el fin de describir los cambios en forma asociados con el aumento de tamaño durante el crecimiento de los individuos se efectuó una regresión multivariada de las coordenadas Procrustes sobre el log del tamaño centroide (TC) como variable independiente. Este modelo asume que las variables independientes constituyen factores que influyen en los cambios en forma y permite determinar la extensión y naturaleza de estos cambios atribuibles, en este caso, al crecimiento (Monteiro, 1999; Monteiro et al., 2005). El modelo de regresión determina, asimismo, la porción de variación en forma explicada por la covariación con las variables independientes (coeficiente de determinación $\mathrm{R}^{2}$ ), calculada a partir de la suma de las distancias Procrustes al cuadrado entre la forma observada y la predicha por el modelo de regresión para cada individuo. Los análisis de regresión se efectuaron para los individuos subadultos y adultos por separado. Los cambios en forma fueron visualizados me- diante la deformación de un modelo 3D de la mandíbula empleando el método de interpolación propuesto por Wiley et al. (2005) y mediante vectores que indican la dirección de cambio de cada landmark (Klingenberg, 2013). Finalmente, se efectuó un análisis de componentes principales a partir de las coordenadas Procrustes de los 4 individuos para los cuales se cuenta con datos histológicos, con el fin de evaluar las diferencias en forma entre los mismos.

Dado que las técnicas de morfometría geométrica permiten cuantificar cambios en el tamaño global de las estructuras anatómicas (registrado como TC) pero no la magnitud del cambio en direcciones particulares, de manera complementaria se obtuvieron medidas lineales entre pares de landmarks con el fin de cuantificar el incremento en tamaño en la longitud y el ancho del cuerpo y la rama mandibular. En la Figura 1 se indican las cuatro medidas lineales uti-
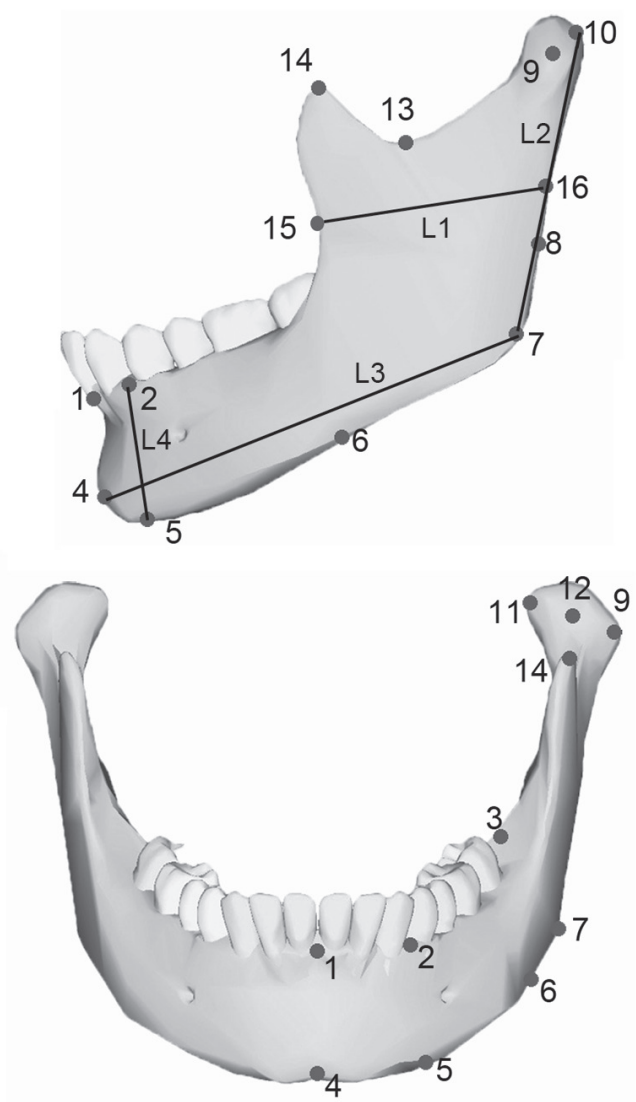

Fig. 1. Landmarks registrados sobre la mandíbula y medidas lineales obtenidas a partir de los mismos. 
lizadas (ver definiciones en Apéndice Online 1). Los cambios en la magnitud de estas variables con la edad se evaluaron mediante un análisis de ANOVA, utilizando las categorías adultosubadulto como variables independientes.

Los análisis morfométricos fueron efectuados empleando los programas MorphoJ 2.0, Landmark y R 3.1.1 (Klingenberg, 2011; Wiley et al., 2005; R Core Team, 2014).

\section{Análisis histológicos}

El presente estudio surge por el interés de integrar la información procedente del análisis de morfometría geométrica y los datos histológicos. Para ello, hemos considerado la información obtenida a partir de la misma muestra en estudios independientes y previos al diseño del presente estudio. Respecto al análisis histológico, los datos proceden de un trabajo previo realizado por una de las autoras (Martinez-Maza et al., 2010) en el que se analizaron las mandíbulas de 12 individuos, 6 subadultos (femeninos $=3$, masculinos $=3$, entre 7 y 17 años) y 6 adultos (femeninos $=3$, masculinos $=3$, entre 24 y 38 años). Para 4 de estos individuos (218 (10 años), 100 (7 años), 126 (8 años), 342 (28 años)) se obtuvieron también datos morfométricos en $3 \mathrm{D}$ y lineales. Las categorías de edad se establecieron considerando, al igual que en los estudios de morfometría geométrica, la emergencia del tercer molar. De este modo, se agruparon los individuos con el tercer molar no emergido o en proceso de emergencia como subadultos, mientras que los adultos son aquellos que tienen el tercer molar completamente emergido. Para la obtención de los datos histológicos, se empleó una técnica no intrusiva mediada por réplicas de alta resolución de la superficie perióstica del hueso (Bromage, 1989; Martinez-Maza et al., 2010) y su posterior análisis con microscopio de luz incidente (Martinez-Maza et al., 2010). Previamente las superficies fueron limpiadas con alcohol al 60\% aplicado con cepillo de cerdas finas y blandas. Posteriormente se realizaron réplicas de la superficie bucal, a partir de un molde negativo realizado en silicona de baja viscosidad (Exaflex injection type 3 low viscosity; DVD Dental SA, España) que copia la superficie ósea. Este molde se prepara delimitando su contorno con una silicona de endurecimiento rápido de modo que actúe como pared de contención (Optosil P plus y Optosil Xantopren; DVD Dental SA, España). A partir de este molde negativo se elaboró un molde positivo en resina epoxi (Feropur-Feroca SA, España). Los moldes de resina fueron metalizados con oro (SC510 BioRad) y se analizaron con un microscopio óptico de luz incidente (Olympus BX51TRF equipado con una cámara digital DP11). Para facilitar el estudio de la superficie del hueso y la localización de las características histológicas identificadas durante el análisis microscópico se graficó sobre la superficie metalizada de la réplica positiva una cuadrícula con cuadrados de $5 \times 5$ $\mathrm{mm}$ utilizando un rotulador permanente fino. A cada cuadrado se le asignó una coordenada $(\mathrm{x}, \mathrm{y})$ de modo que el origen se encuentra en el cuadrado inferior izquierdo $(1,1)$. Se identificaron en la superficie de la mandíbula los rasgos microestructurales relacionados con los procesos de formación y reabsorción ósea. Las superficies de formación son el resultado de la actividad de los osteoblastos y están caracterizadas por la presencia de paquetes de fibras de colágeno, mientras que las superficies de reabsorción son el producto de la actividad de los osteoclastos que degradan y reabsorben el hueso, generando concavidades denominadas lagunas de Howship (Boyde, 1972; Bromage 1984, 1989; Martínez Maza et al., 2010). El registro de estos campos de formación y reabsorción permitió establecer un mapa mostrando su distribución, que recibe el nombre de Patrón de Modelado Óseo (PMO).

\section{RESULTADOS}

Los resultados del análisis de regresión realizado a partir de los individuos subadultos indican que los cambios alométricos en el periodo de 7 a 18 años son significativos y el tamaño da cuenta del $8,4 \%$ de la variación en las coordenadas de forma (Figs. 2 y 3). El aumento de tamaño con el crecimiento se asocia a cambios en forma caracterizados, especialmente, por un desplazamiento en sentido craneal (o superior) del cóndilo, la escotadura, la apófisis coronoides y la sínfisis mandibular, y un desplazamiento en sentido caudal (o inferior) del gonion y el borde posterior de la rama (Fig. 2). Como resultado de estos cambios, la morfología de la rama mandibular en los individuos de 

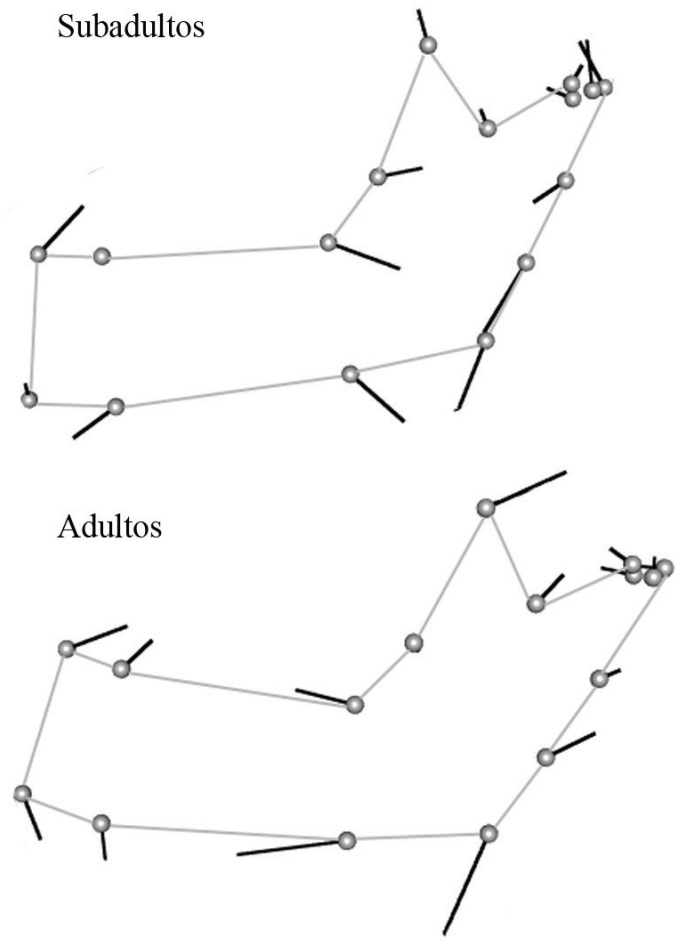

Fig. 2. Cambios en forma obtenidos a partir de la regresión de las coordenadas superpuestas sobre el tamaño centroide. Los vectores muestran la dirección y magnitud de los cambios asociados al aumento de tamaño en individuos subadultos y adultos. Los puntos representan la forma consenso. mayor tamaño se caracteriza por un incremento en la altura relativa y un menor ancho relativo con respecto a las mandíbulas de menor tamaño (Fig. 3). Asimismo, se observa una modificación del ángulo entre la rama y el cuerpo mandibular, reduciéndose a medida que aumenta el TC. En vista superior la forma general de la mandíbula varía entre morfologías en forma de $\mathrm{U}$ y de $\mathrm{V}$ de acuerdo con el incremento en tamaño de los individuos (Fig. 3). Es importante aclarar que los cambios en forma observados en subadultos y adultos deben ser interpretados con relación al conjunto de mandíbulas analizadas dentro de cada categoría. Es decir, los individuos subadultos de mayor tamaño presentan morfologías relativamente más estrechas que los individuos subadultos de menor tamaño. Al analizar los adultos se observa que los individuos de menor tamaño presentan mandíbulas más redondeadas en relación con los adultos de mayor tamaño (no en relación con los subadultos).

El análisis de regresión realizado sobre los individuos adultos muestra que, a diferencia de lo observado en los subadultos, el TC explica un porcentaje muy reducido $(2,6 \%)$ y no significativo de la variación en forma. El patrón de cambio en forma asociado al tamaño en individuos mayores a 18 años difiere levemente del observado en los subadultos (Figs. 2 y 3), aunque en líneas

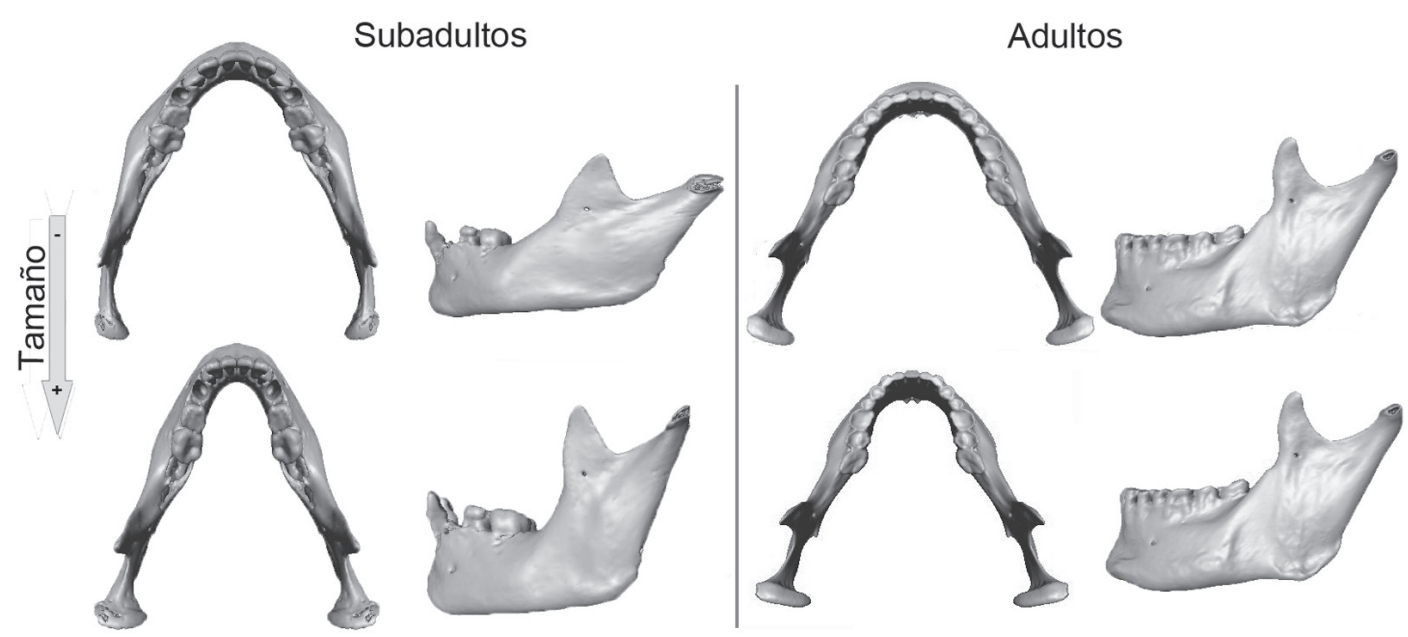

Fig. 3. Variación en forma asociada al tamaño obtenida a partir de regresiones en los individuos subadultos y adultos. Las superficies de las mandíbulas representan los cambios de forma observados entre individuos de menor y mayor tamaño con respecto al consenso de cada grupo (subadultos y adultos). 
generales se observa también el desplazamiento craneal de la parte superior de la rama y el desplazamiento caudal de los landmarks ubicados en el borde inferior de la mandíbula.

La figura 4 muestra los cambios en las medidas lineales asociados con la edad de los individuos. Se observa que la altura de la rama y la longitud del cuerpo mandibular presentan cambios marcados hasta los 18 años, y se mantienen relativamente estables a partir de esa edad (Fig. 4). La altura del cuerpo exhibe un patrón similar de incremento con la edad, aunque menos marcado. Por el contrario, no se observaron cambios notables en el ancho de la rama entre subadultos y adultos (Fig. 4). Los resultados de
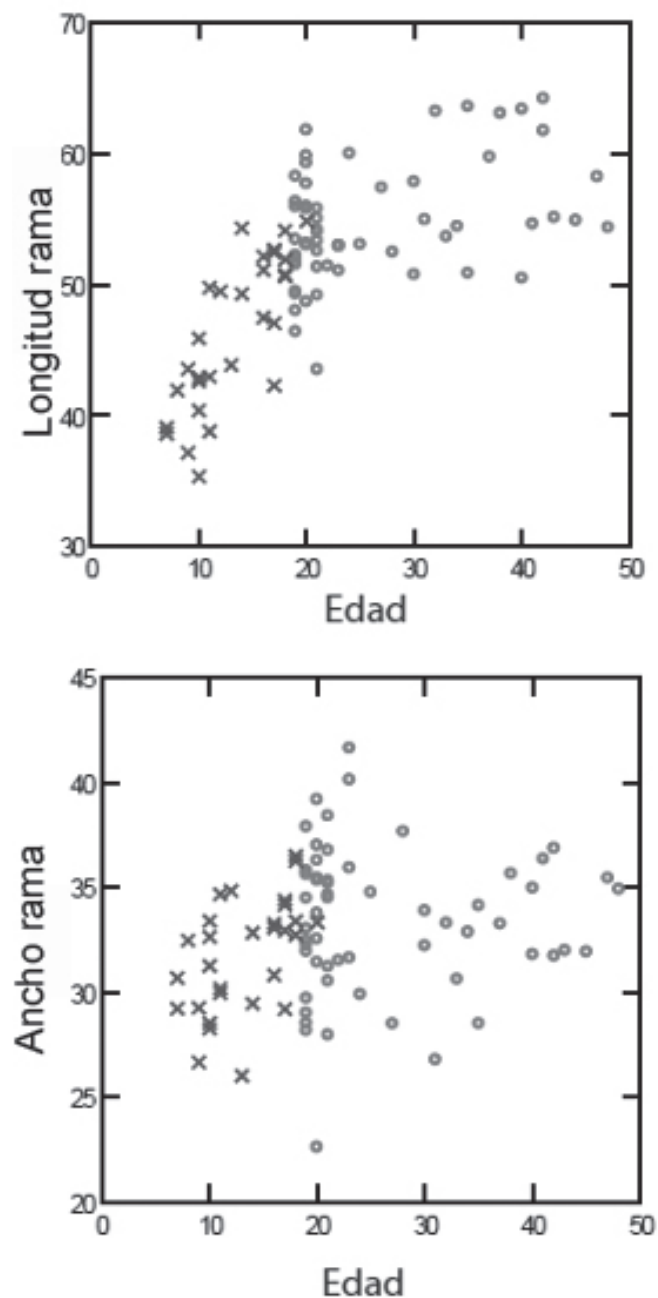

la prueba ANOVA confirman la existencia de diferencias significativas entre las categorías subadultos y adultos para la altura de la rama $\left(\mathrm{F}_{1-85}=55,15 ; \mathrm{p}<0,001\right)$, la longitud del cuerpo $\left(\mathrm{F}_{1-85}=28,01 ; \mathrm{p}<0,001\right)$ y la altura del cuerpo $\left(\mathrm{F}_{1-85}=9,69 ; \mathrm{p}=0,002\right)$, mientras que no se detectaron diferencias altamente significativas para el ancho de la rama $\left(\mathrm{F}_{1-85}=4,88 ; \mathrm{p}=0,03\right)$.

La figura 5 muestra la variación en forma de los cuatro individuos para los que se cuenta con datos morfométricos e histológicos. Los 3 individuos subadultos exhiben diferencias en forma. En particular, se destaca el individuo 100 por presentar un menor desarrollo de la rama mandibular con respecto al cuerpo. El individuo 218 presenta una
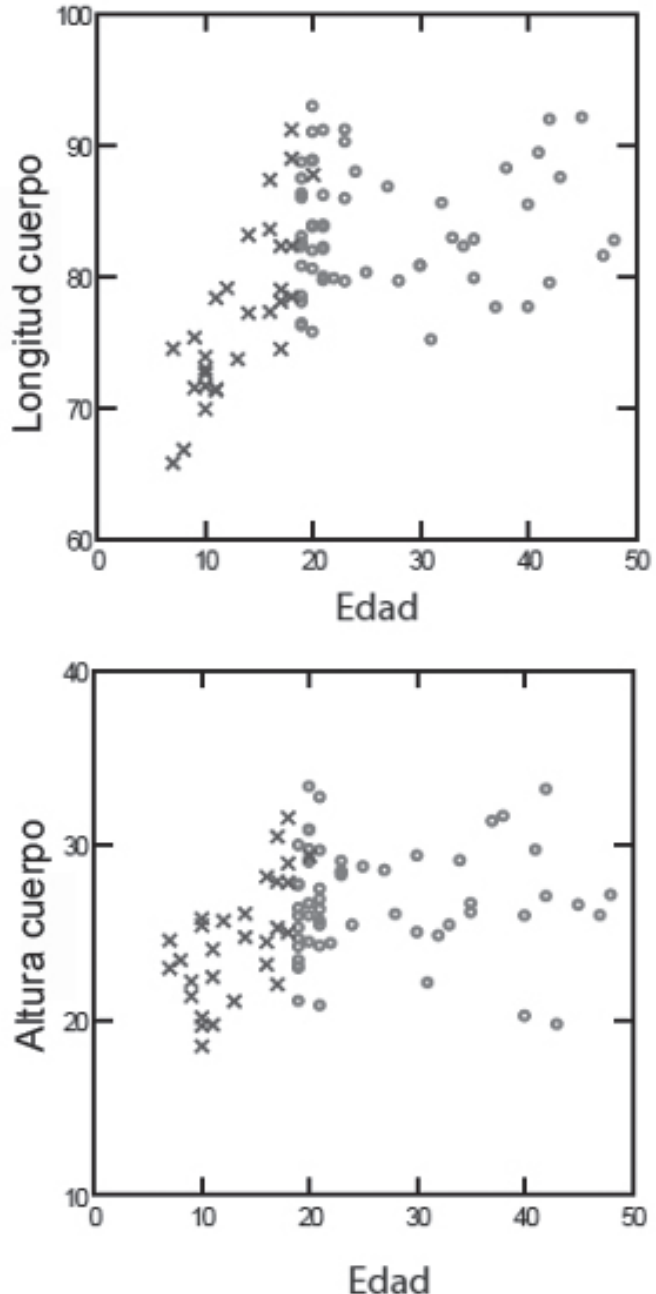

Fig. 4. Medidas de distancia lineales vs. edad. Las cruces indican los individuos subadultos y los círculos, los adultos. 


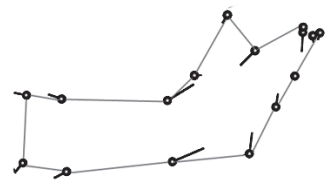

100
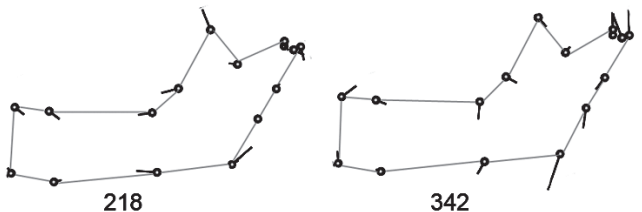

Fig. 5. Diferencias de forma entre la configuración consenso y los individuos 100, 126, 218 y 342. Las esferas representan la forma consenso y los vectores los desplazamientos correspondientes a cada individuo.

menor longitud relativa del cuerpo y de la rama con respecto a la forma consenso, mientras el individuo adulto (342) se destaca la mayor longitud relativa de la rama con respecto a la forma consenso.

El análisis de los PMO individuales de 6 subadultos y 6 adultos, permitió establecer el patrón general que muestra los campos comunes en cada grupo de edad (Fig. 6a). Las direcciones de crecimiento inferidas a partir de estos PMO generales y su interpretación en el marco teórico de la biología craneofacial indica que el crecimiento de la mandíbula ocurre principalmente por formación de hueso en la parte posterior del cuerpo y de la rama mandibular con reabsorción en la parte anterior de la rama (e.g. Enlow y Harris, 1964; Enlow y Hans, 1996). Esto conlleva a la reubicación posterior de la mandíbula y el crecimiento del cuerpo en su parte posterior. Simultáneamente ocurre un desplazamiento anterior e inferior de toda la mandíbula que implica a su vez el crecimiento en altura de la rama mandibular para mantener el contacto con el cráneo a través de la articulación temporomandibular. Estas dinámicas se relacionan con el incremento en la longitud del cuerpo mandibular tanto en la región sinfisaria como en su parte posterior mientras la rama se reubica en una posición posterior. La rama mandibular muestra también un crecimiento superior en la región del cóndilo que participaría en el incremento en altura de esta región manteniendo el contacto con la base del cráneo a través de la articulación temporomandibular. Simultáneamente, la mandíbula mostraría un crecimiento lateral del cuerpo, un crecimiento medial de la región de la apófisis coronoides y un crecimiento lateral de la región posterior de la rama.

En los adultos se observa un cambio en el PMO que refleja diferencias en las dinámicas de crecimiento de la mandíbula. Se observa un incremento de la reabsorción en la región de contacto cuerpo-rama y en la rama mandibular que indica un marcado crecimiento medial de esta región mandibular. Simultáneamente, la mandíbula muestra un crecimiento anterior y lateral del cuerpo mientras el cóndilo crece hacia arriba manteniendo el contacto con la base del cráneo. De manera particular, hay que destacar las dinámicas de la región sinfisaria tanto en los subadultos como en los adultos. La presencia del campo de reabsorción en el componente alveolar característico de los humanos modernos refleja la proyección del componente basal que subyace a la formación del mentón, rasgo distintivo de nuestra especie.

La figura $6 \mathrm{~b}$ muestra los patrones de modelado óseo individuales obtenidos de los individuos para los cuales se cuenta con datos morfométricos e histológicos. Se trata de una muestra pequeña que ha sido seleccionada a los fines de realizar un estudio preliminar para explorar la integración de datos histológicos y de morfometría geométrica. Los PMO individuales muestran un mosaico de campos de formación y reabsorción de hueso de tamaño y forma variable que se distribuyen de forma particular en cada mandíbula. En el grupo de los subadultos, observamos dos mandíbulas (126 y 218) con un patrón similar caracterizado por campos de formación de hueso con campos de reabsorción localizados en el componente alveolar de la región sinfisaria. Además, el individuo 126, con mayor conservación de la superficie ósea, muestra campos de reabsorción en la región de la apófisis coronoides y en el cuello del cóndilo. El individuo 100 también presenta un campo de reabsorción en el componente alveolar de la región sinfisaria. Sin embargo, este individuo muestra un predominio de los campos de reabsorción en la superficie bucal de la rama al contrario de lo observado en los dos individuos descritos anteriormente. La mandíbula del individuo adulto (342) se caracteriza por la presencia de campos de formación de hueso con campos de reabsorción localizados en la zona de contacto cuerpo-rama y en la región posterior de la rama. 
a

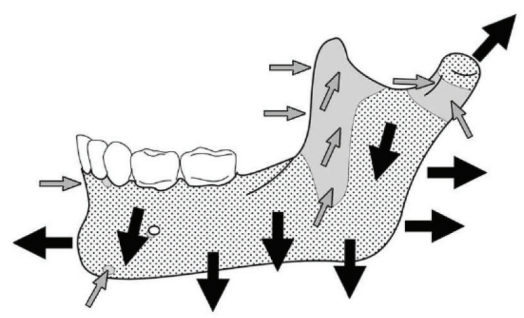

b

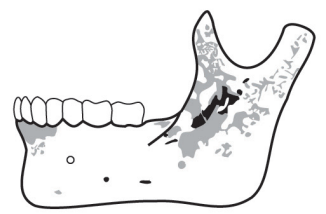

100 (7 años)

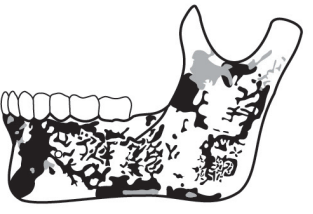

126 (8 años)
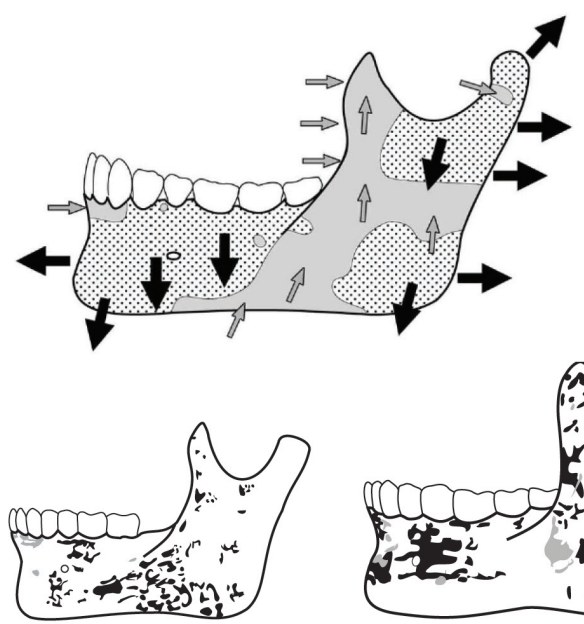

218 (10 años)

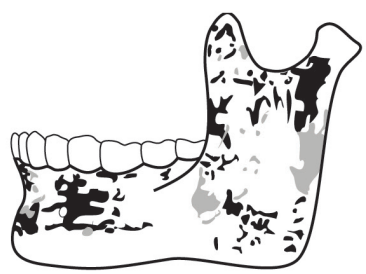

342 (28 años)

Fig. 6. Patrones de modelado óseo y direcciones de crecimiento inferidas. a: Patrones de modelado óseo generalizados (Izquierda: subadultos, Derecha: adultos). b: Patrones de modelado óseo de los cuatro individuos incluidos en este estudio. Estos patrones muestran la distribución de los campos de modelado en el lado bucal de la mandíbula de individuos subadultos (126, 100 y 218) y adultos (342). Las flechas negras indican la dirección del crecimiento y las flechas grises indican la dirección opuesta al crecimiento debido a la eliminación de hueso. Modificado a partir de Martínez-Maza et al. (2013).

\section{DISCUSIÓN}

\section{Cambios morfológicos en la mandíbula durante el desarrollo}

El estudio preliminar que hemos realizado nos ha permitido observar que tanto los datos morfométricos como los histológicos indican que durante el crecimiento se produce un incremento en altura de la región sinfisaria y el cuerpo mandibular (Fig. 4). Asimismo, ambos análisis han mostrado un incremento en altura de la rama mandibular, que en el caso de los datos histológicos quedaría reflejado en las dinámicas inferidas en la región del cóndilo. Junto con el aumento en altura, el crecimiento de la mandíbula muestra un aumento en la longitud del cuerpo (Figs. 2 y 4). Considerando las direcciones de crecimiento inferidas a partir de los PMO de los subadultos y adultos, este incremento en longitud ocurre por la reubicación de la rama en una posición posterior -marcado por la reabsorción en el borde anterior y formación en el borde posterior- de modo que el cuerpo mandibular aumenta su longitud en la región posterior (contacto cuerpo-rama) y crece en la región sinfisaria.
Uno de los resultados más interesantes que se han obtenido con los análisis de morfometría geométrica es la rotación hacia arriba (upward) de la mandíbula durante el desarrollo. Los datos morfométricos muestran un movimiento superior de la parte anterior de la mandíbula y un movimiento inferior en la parte posterior del cuerpo y de la rama mandibular. Estos resultados coinciden con los descritos por Bastir et al. (2007) al analizar los cambios ontogenéticos en la forma de la mandíbula en humanos modernos empleando dos muestras europeas de los siglos XIX y XX (Coimbra, Portugal; Spitalfields, Reino Unido). El movimiento de rotación observado en el análisis morfométrico no se puede inferir a partir de los datos histológicos, sin embargo, podemos plantear hipótesis sobre cómo ocurre esa rotación partiendo de los PMO. Si tenemos en cuenta el crecimiento descrito anteriormente, para que la mandíbula muestre una rotación hacia arriba tiene que ocurrir que el incremento en altura de la rama sea mayor que el incremento en altura de la región anterior de la mandíbula. Los PMO permiten inferir la dirección del crecimiento pero no la tasa a la que crece el hueso en dicha dirección. De este modo, la integración de la histología con la morfometría geométrica 
es fundamental para comprender cómo ocurren determinados movimientos que sólo se detectan mediante análisis morfométricos.

Este conjunto de dinámicas de crecimiento coincide con datos de trabajos previos que describen en la mandíbula humana un fuerte vector de crecimiento posterior y una ligera rotación hacia delante (Enlow y Harris, 1964; Moss y Salentijn, 1970; Björk y Skieller, 1983; Enlow y Hans, 1996). La rotación de la mandíbula se ha relacionado con la rotación de la maxila (upward maxillary rotation, airorhynchy) que da como resultado la rotación hacia arriba del plano oclusal. Esta rotación se compensa mediante dinámicas de crecimiento en la maxila y mediante la deriva cortical inferior del paladar (Moss y Salentijn, 1970; Björk y Skieller, 1983; Bromage, 1989; Enlow y Hans, 1996; McCollum y Ward, 1997; Martinez-Maza et al., 2013).

\section{Comparación inter-individual en sub- adultos}

Los PMO de los tres individuos subadultos muestran similitudes en el cuerpo mandibular y la región sinfisaria relacionadas con el predominio de campos de formación y la presencia de un campo de reabsorción en la región alveolar sinfisaria (Fig. 6b). Este patrón es similar al obtenido en trabajos previos por Enlow y colaboradores (Enlow yHans, 1996; Kurihara et al., 1980). Sin embargo, hemos observado diferencias con respecto al PMO de la rama mandibular de modo que los individuos 126 y 218 presentan un patrón similar al establecido por Enlow y Hans (1996) mientras que el individuo 100 presenta un PMO diferente y caracterizado por reabsorción ósea (Fig. 6b). Los análisis de morfometría geométrica también han mostrado diferencias inter-individuales (Fig. 5) que podrían estar relacionadas con las diferencias en la distribución de los campos de modelado óseo. La rama de los individuos 126 y 218 presenta un PMO caracterizado por un predominio de los campos de formación ósea así como reabsorción en la región superior (ver individuo 126) que indicaría un crecimiento predominantemente lateral y el crecimiento medial de la región superior de la rama. Tanto el PMO como las direcciones inferidas coinciden con el modelo de crecimiento de la rama propuesto por Enlow y Hans (1996) y
Kurihara et al. (1980). Por el contrario, el predominio de superficies de reabsorción en la mandíbula del individuo 100 indica un crecimiento medial pronunciado.

Asimismo, los datos morfométricos muestran diferencias entre el individuo 100 y los otros dos individuos que podrían estar relacionadas con la reubicación posterior de la mandíbula y el incremento en longitud del cuerpo mandibular así como un marcado incremento de la altura de la rama. Considerando en conjunto los datos histológicos y morfométricos, y teniendo en cuenta el marco teórico de biología craneofacial (Moss, 1962; 1997a-d; Enlow y Hans, 1996) planteamos que la diferencia en las dinámicas de crecimiento de la rama del individuo 100 podría ser resultado de una reubicación posterior de la rama más pronunciada y un crecimiento medial de esta región. Las diferencias en el modelado óseo de la rama mandibular han sido descritas en trabajos previos los cuales han propuesto que dicha variabilidad podría expresarse en diferencias morfológicas (Hans et al., 1995; Martinez-Maza et al., 2013). La aproximación metodológica seguida en este trabajo nos ha permitido valorar este punto $\mathrm{y}$, si bien la muestra es limitada, los resultados apoyarían dicha hipótesis.

Por otro lado, hemos observado diferencias a nivel de la región sinfisaria. Los individuos muestran un patrón similar al PMO general de esta región que coincide con el patrón descrito por Enlow y Hans (1996) y que se caracteriza por la formación ósea y reabsorción en el componente alveolar. Sin embargo, el individuo 126 muestra un predominio de los campos de formación de hueso en el componente alveolar de la región sinfisaria mientras que en los individuos 100 y 218 la actividad de reabsorción de hueso es mayor que la de formación. Enlow y Harris (1964) mostraron que el PMO de la región sinfisaria presenta una gran variabilidad respecto a la extensión del campo de reabsorción que podría estar relacionada con diferentes morfologías. En este marco, el patrón observado en los individuos 100 y 218 podría relacionarse con el desarrollo y proyección del mentón, mientras que el patrón del individuo 126 indicaría un crecimiento hacia delante de toda la cara bucal de la región sinfisaria. A pesar de que los datos histológicos muestran variación con respecto al campo de reabsorción de la región alveolar 
(Fig. 6b), los datos morfométricos indican que las diferencias entre el individuo 126 y los otros dos individuos se localiza en el componente basal (Fig. 5). ¿Cómo podemos explicar la variación morfológica de una región que muestra las mismas dinámicas de crecimiento entre individuos? Nuestros datos indican que la variación morfológica se debe a un aspecto del crecimiento distinto al tipo de actividad de modelado óseo. Como hemos indicado anteriormente, los PMO permiten inferir las direcciones de crecimiento pero no la tasa a la que crece en dicha dirección. Por lo tanto, podemos plantear que las diferencias morfológicas de la región sinfisaria podrían responder a distintas tasas de formación ósea en el componente basal.

\section{CONCLUSIÓN}

En resumen, los resultados del análisis conjunto de los patrones de variación en tamaño y forma, y de remodelado óseo a lo largo de la ontogenia de la mandíbula en Homo sapiens indican que los cambios a escala anatómica descritos mediante técnicas morfométricas fueron, en líneas generales, concordantes con los inferidos a partir de la distribución de las áreas de formación y reabsorción ósea. Algunos cambios importantes vinculados con el movimiento de rotación del cuerpo con respecto a la rama mandibular sólo pudieron detectarse a través del análisis morfométrico. En la comparación inter-individual se detectaron diferencias en las direcciones de cambio en la sínfisis mandibular derivadas de los PMO y la variación en forma registrada con las técnicas morfométricas. Esto sugiere que la información provista por las superficies de formación y reabsorción ósea acerca de los mecanismos que subyacen a la dinámica de crecimiento es parcialmente transferida y retenida en la escala anatómica. En conclusión, la integración de los datos que proceden de la histología y de la morfometría permite complementar el análisis de los movimientos y las direcciones de crecimiento que no pueden ser inferidas a partir de cada una de estas fuentes de información independientemente, de modo que podamos comprender cómo ocurren los cambios morfológicos durante el desarrollo y cómo se originan las diferencias inter-individuales.

\section{AGRADECIMIENTOS}

A Ana Luisa Santos y el personal del Museo Antropológico de Coimbra (Portugal) por permitir el acceso a la Colección de Esqueletos Identificados allí depositada.

\section{LITERATURA CITADA}

Adams DC, Rohlf FJ, Slice DE. 2004. Geometric morphometrics: ten years of progress following the revolution>. Ital J Zool 71:5-16.

Atchley WR, Hall BK. 1991. A model for development and evolution of complex morphological structures. Biol Rev Camb Philos Soc 66(2):101-57.

Bastir M, O’Higgins P, Rosas A. 2007. Facial ontogeny in Neanderthals and modern humans. Proc Biol Sci 274(1614):1125-1132. doi:10.1098/rspb.2006.0448

Björk A, Skieller V. 1983. Normal and abnormal growth of the mandible. A synthesis of longitudinal cephalometric implant studies over a period of 25 years. Eur J Orthod 5:1-46.

Boyde A. 1972. Scanning electron microscope studies of bone. En: Bourne GH editor. The biochemistry and physiology of bone. New York: Academic Press. p. 259-310.

Brachetta Aporta N, Martinez-Maza C, Gonzalez P, Bernal V. 2014. Bone modeling patterns and morphometric creaniofacial variation in individuals from two prehistoric human populations from Argentina. Anat Rec 297:1829-1838. doi:10.1002/ar.22999

Bromage TG. 1984. Surface remodelling studies on fossil bone. J Dent Res 63:491.

Bromage TG. 1989. Ontogeny of the early human face. J Hum Evol 18:751-773.

Enlow DH. 1963. Principles of bone remodelling. Springfield: CC Thomas Publisher.

Enlow DH. 1966. A comparative study of facial growth in Homo and Macaca. Am J Phys Anthropol 24:293-308.

Enlow DH. 1982. Handbook of facial growth. Philadelphia: WB Saunders Company

Enlow DH, Hans MG. 1996. Essentials of facial growth. Philadelphia: WB Saunders Company.

Enlow DH, Harris DB. 1964. A study of postnatal growth of the human mandible. Am J Orthod 50:25-50.

Freidline SE, Martinez-Maza C, Hublin JJ. 2014. An integrative approach to studying craniofacial development in great apes and humans. Am J Phys Anthropol S58:121. doi:10.1002/ajpa.22488

Halazonetis DJ. 2007. Morphometric correlation between facial soft-tissue profile shape and skeletal pattern in children and adolescents. Am J Orthod Dentofacial 132:450-457. doi:10.1016/j.ajodo.2005.10.033

Hall BK. 2003. Unlocking the black box between genotype and phenotype: Cell condensations as morphogenetic (modular) units. Biology and Philosophy 18 (2):219-247.

Hans MG, Enlow DH, Noachtar R. 1995. Age-related differences in mandibular ramus growth: a histologic study. Angle Orthod 65:335-340.

Klingenberg CP. 2011. MorphoJ: an integrated software package for geometric morphometrics. Mol Ecol Resour 11:353-357.

Klingenberg CP. 2013. Visualizations in geometric morphometrics: how to read and how to make graphs showing shape changes. Hystrix 24:15-24. 
Kurihara S, Enlow DH, Rangel RD. 1980. Remodeling reversals in anterior parts of the human mandible and maxilla. Angle Orthod 50:98-106.

Martinez-Maza C, Rosas A, Nieto-Diaz M. 2010. Identification of bone formation and resorption surfaces by reflected light microscopy. Am J Phys Anthropol 143:313-320. doi:10.1002/ajpa.21352

Martinez-Maza C, Rosas A, Nieto-Diaz M. 2013. Postnatal changes in the growth dynamics of the human face revealed from the bone modelling patterns. J Anat 223:228-241. doi:10.1111/joa.12075

McCollum MA, Ward SC. 1997. Subnasoalveolar anatomy and hominoid phylogeny: evidence from comparative ontogeny. Am J Phys Anthropol 102:377-405. doi:10.1002/ (SICI) 1096-8644(199703)102:3<377::AIDAJPA7>3.0.CO;2-S

Mitteroecker P, Gunz P, Bernhard M, Schaefer K, Bookstein FL. 2004. Comparison of cranial ontogenetic trajectories among great apes and humans. J Hum Evol 46:679698. doi:10.1016/j.jhevol.2004.03.006

Mitteroecker P, Gunz P. 2009. Advances in Geometric Morphometrics. J Evol Biol 36:235-247. doi:10.1007/ s11692-009-9055-X

Monteiro LR. 1999. Multivariate regression models and geometric morphometrics: the search for causal factors in the analysis of shape. Syst Biol 48:192-199.

Monteiro LR, Bonato V, Dos Reis SF. 2005. Evolutionary integration and morphological diversification in complex morphological structures: mandible shape divergence in spiny rats (Rodentia, Echimyidae). Evol Dev 7 (5):429-439. doi:10.1111/j.1525-142X.2005.05047.x

Moss ML. 1962. The functional matrix. En: Kraus BS, Riedel RA editores. Vistas in Orthodontics. Philadelphia: Lea and Febiger. p. 85-98.

Moss ML. 1970. Enamel and bone in shark teeth: with a note on fibrous enamel in fishes. Acta Anat 77:161-187.

Moss ML. 1997a. The functional matrix hypothesis revis- ited. 1. The role of mechanotransduction. Am J Orthod Dentofacial Orthop 112:8-11.

Moss ML. 1997b. The functional matrix hypothesis revisited. 2. The role of an osseus connected cellular network. Am J Orthod Dentofacial Orthop 112:221-226.

Moss ML. 1997c. The functional matrix hypothesis revisited. 3. The genomic thesis. Am J Orthod Dentofacial Orthop 112:338-342.

Moss ML. 1997d. The functional matrix hypothesis revisited. 4. The epigenetic antithesis and the resolving synthesis. Am J Orthod Dentofacial Orthop 112:410-417.

Moss ML, Salentijn L. 1970. The logarithmic growth of the human mandible. Acta Anat 77:341-360.

O'Higgins P, Jones N. 1998. Facial growth in Cercocebus torquatus: an application of three-dimensional geometric morphometric techniques to the study of morphological variation. J Anat 193 (2):251-272. doi:10.1046/ j.1469-7580.1998.19320251.x

R Core Team. 2014. R: A language and environment for statistical computing. R Foundation for Statistical Computing, Vienna, Austria. URL http://www.R-project.org/

Rocha MA. 1995. Les collections ostéologiques humaines identifiées du Musee Anthropologique de l'Université de Coimbra. Antropologia Portuguesa 13:7-39.

Rohlf FJ, Slice DE. 1990. Extensions of the procrustes method for the optimal superimposition of landmarks. Syst Zool 39:40-59. doi:10.2307/2992207

Wiley DF, Amenta N, Alcantara DA Ghosh D, Kil YJ, Delson E, Harcourt-Smith W, Rohlf FJ, St John K, Hamann B. 2005. Evolutionary morphing. En: Silva CT, Groeller E, Rushmeier HE, editores. IEEE Visualization 2005, IEEE Los Alamitos, California: Computer Society Press. p 431-438.

Wilson LAB, Ives R, Cardoso HFV, Humphrey LT. 2015. Shape, size, and maturity trajectories of the human ilium. Am J Phys Anthropol 156:19-34. doi:10.1002/ ajpa. 22625 